@Mishra D, Chattopadhyay A, Kavanal AJ, Kumar R, Sharma SK.

\title{
Complex Regional Pain Syndrome
}

\section{Debashish Mishra $^{1}$ (D), Arghya Chattopadhyay' (iD), Anwin Joseph Kavanal' ${ }^{2}$ (D), Rajender Kumar ${ }^{2}$ (iD, Shefali K. Sharma ${ }^{1}$}

${ }^{1}$ Clinical Immunology and Rheumatology Unit, and ${ }^{2}$ Department of Nuclear Medicine, Post Graduate Institute of Medical Education and Research, Chandigarh, India

Mediterr J Rheumatol 2021;32(2):174-5

https://doi.org/10.31138/mjr.32.2 174

Article Submitted: 25 Aug 2020; Revised Form: 8 Dec 2021; Article Accepted: 16 Jan 2021; Available Online: 30 Jun 2021

Keywords: Complex regional pain syndrome, CRPS, reflex sympathetic dystrophy, causalgia, triphasic bone scan

A 63-year-old woman presented with history of severe diffuse pain (even on touch) and swelling over right forearm and hand for 6 weeks. On inquiry, she gives history of past herpetic lesions over same side 4 months back. On examination, there was diffuse swelling, tenderness and warmth over right hand and forearm, along with increased sweating and loss of hair (Figure 1A). Radiograph showed patchy osteopenia around right wrist and small joints of hand (Figure 1B). Triple phase skeletal scintigraphy showed increased flow, perfusion and osteoblastic activity in right shoulder, elbow, wrist, and small joints of hand suggestive of complex regional pain syndrome (CRPS) (Figure 1 C-F). Her inflammatory markers (erythrocyte sedimentation rate and C-reactive protein) were normal, and rheumatoid factor, anti-nuclear antibody, and viral serologies (Human immunodeficiency virus, hepatitis B and C) were negative. Based on clinical and imaging findings, a diagnosis of CRPS triggered by herpes zoster infection was made. She was started on oral bisphosphonates along with non-steroidal

\section{Corresponding Author:}

Shefali K. Sharma

Additional Professor

Clinical Immunology and

Rheumatology Unit

Post Graduate Institute of Medical

Education and Research

Chandigarh, India

Tel.: +919417372439

E-mail: sharmashefali@hotmail.com fuse severe burning pain with difficulty in movements, increased sweating, and vasomotor features like warmth and redness. Radiography and bone scan can aid in diagnosis, depending on the stage of disease. Treatment consists of analgesics, steroids, bisphosphonates, antidepressants, neuropathic medications, sympathetic blockade, spinal cord stimulation and dorsal root ganglion stimulation. However, to prevent CRPS by early mobilisation and graded physiotherapy along with psychological support is more beneficial than treatment., ${ }^{1,2,3}$

\section{CONFLICT OF INTEREST}

The authors declare no conflict of interest.

\section{CONSENT}

Written consent has been obtained from the patient for the publication.

\section{REFERENCES}

1. Gorodkin R. Complex Regional Pain Syndrome (reflex sympathetic dystrophy). In: Hochberg MC, Gravallese EM, Silman AJ, Smolen JS, Weinblatt ME, Weisman MH, $7^{\text {th }}$ ed. Rheumatology, Vol. 1. Philadelphia, PA: Elsevier, 2019:729-35.

2. Misidou C, Papagoras C. Complex Regional Pain Syndrome: An update. Mediterr J Rheumatol 2019 Mar 28;30(1):16-25.

3. Bussa M, Mascaro A, Cuffaro L, Rinaldi S. Adult complex regional pain syndrome type I: A narrative review. PM R 2017;9:707-19. 

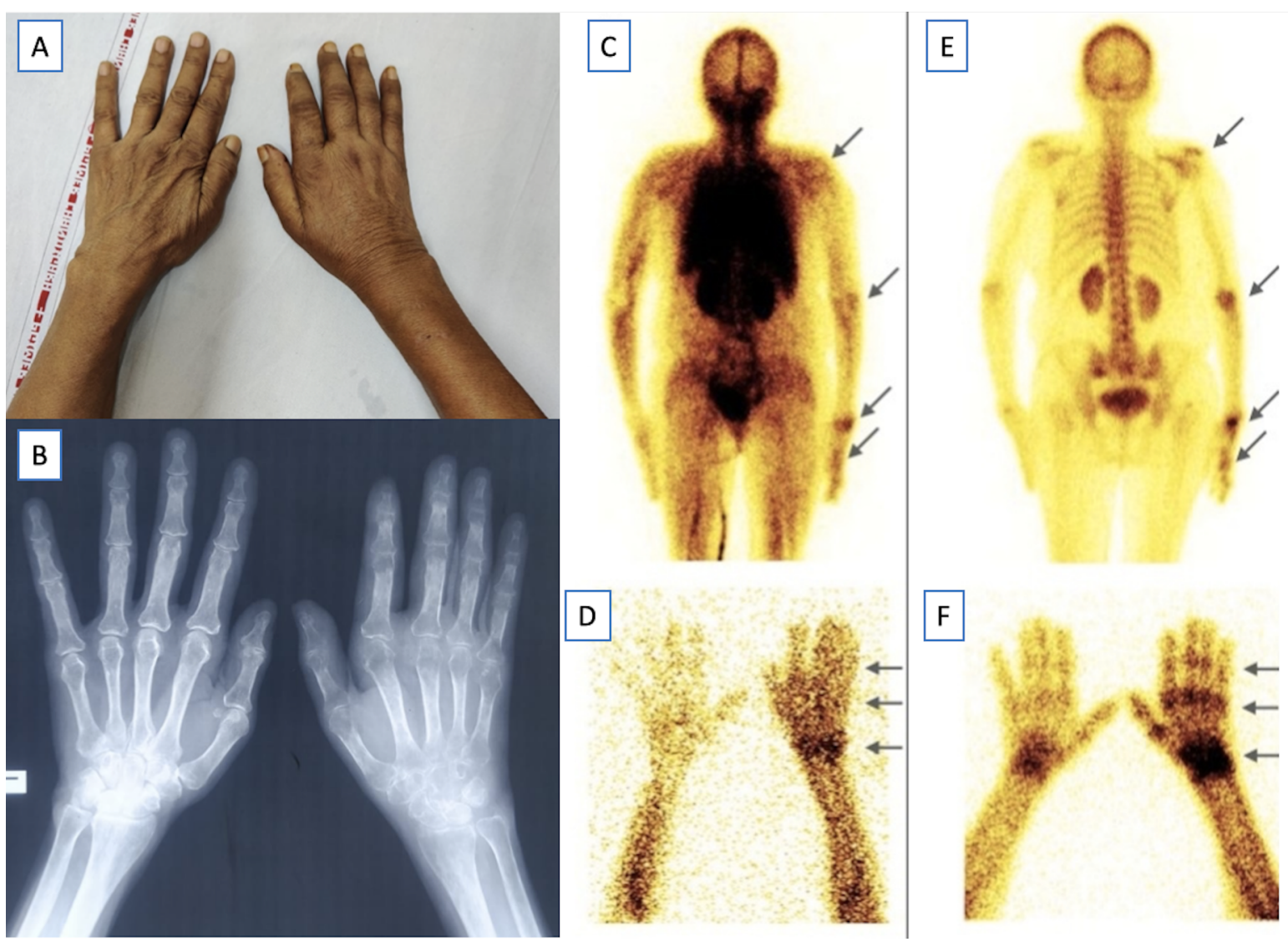

Figure 1: Diffuse swelling over right hand and forearm along with sparse hair (A), X-ray bilateral hands

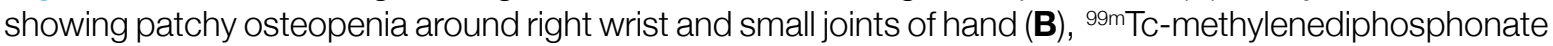
(MDP) three phase bone scan: Blood pool phase images of whole body posterior view $(\mathbf{C})$ and regional hand static dorsal view (D) showing relatively increased pooling of the tracer in the small joints of the right hand, right wrist, right elbow and right shoulder joint regions (arrows) compared to the left side; Delayed phase images of whole body posterior view $(\mathbf{E})$ and regional hand static dorsal view $(\mathbf{F})$ showing relatively increased uptake of the tracer (osteoblastic activity) in the periarticular regions of small joints of the right hand, right wrist, right elbow and right shoulder joint (arrows) compared to the left side. Image findings are suggestive of complex regional pain syndrome (CRPS) involving the right upper limb. 